PRESIDENT: W. Livingston

Commission Meeting, 26 November 1985

Dr. Colin M. Humphries was elected the new President. For Vice-President there were two nominations with John Davis having the winning vote. The following members of the Organizing Committee were slated and approved: I. C. Bhat tacharyya, J. Davis, O. Engvold, B. Fort N. Hid, W. Livingston (Retiring Fresident), D. Malin, N. Steshenko, R. Tull, G. Walker, W. L. Wilcock, and G. Wlerick. A list of new applications for membership in the Commission was submitted and approved.

Brief summaries of bisiness in the Working Groups were presented by their Chairman. It was noted that Dr. Elizabeth M. Sims retired as Chairperson of Astronomical Photography WG, being replaced by Dr. David Malin. Dr. John Davis continues as Chairman of the High Angular Resolution Interferometry WG, and Dr. Gordon Walker has accepted Chairmanship of the Detector WG.

Dr. J. Tinbergen, President of Commission 25, presented a Resolution on Instrumental Polarization for our consideration. Calling attention to the fact that several propused designs for large telescopes in the fixture incorporate severe oblique reflections, he pointed out that this feature will virtually exclude precision polarimetry. He urges designers to avoid such oprical schemes where possible. Our membership unanimously approved Tinbergen's resulution al though it was too late in the General Assembly for of ficial recognition of this vote.

Special Science Report: First Light at India's $2.3 \mathrm{~m}$ Telescope

Professor J. C. Bhattacharyya, India Institute of Astrophysios, beamed as he circulated first-light pictures taken at the prime focus of the $2.34 \mathrm{~m}$ telescope at Kavalur. The star images proved first rate and India can well be proud of this achievement by indigenous skills. Mr. A. P. Jayarajan, project optican, and $\mathrm{Mr}$. A. Tapde, mechanical engineer, described the history and special features of the telescope.

This project was the brainchild and dream of one individual, the late Dr. M. K. Vainu Bappu. It was he who visualized and practically designed the entire instrument. No detail was beyond his concern. Some milestones include: Funding provided 1973, mirror blank received and engineering started 1974, housing completed 1978, mirror grinding began 1979, dome installed 1981, mechanical mount shop tested 1984, and 'first light' October 31, 1985.

Four foci are available: the prime focus at $\mathrm{f} / 3.25$, the cassegrain at $\mathrm{f} / 13$, and coude at $\mathrm{f} / 43.25$. A 'Zerodur' blank was obtained from Schott which, after grinding, has a thickness diameter ratio of $1 / 6$. During figuring two tests proved both convenient and precise. The 'axial wire test' was emplyed daily complemented by the 'Abe of ner null corrector test' especially towards the final stages of figuring. Some local zonal errors persist but the accuracy of the entire surface is better than a tenth of a wave. Cut-off measurements by a Foucault knife edge indicate that $90 \%$ of the imaged light falls within 0.6 arc sec. 
The telescope housing is ample: $23 \mathrm{~m}$ in diameter with the declination axis $18.5 \mathrm{~m}$ above ground. The telescope itself has an equatorial horse-shoe yoke configuration with hydrostatic bearings. Drives, both RA and Dec, employ 2.8-m bull gears with two pinions for antibacklash. The final cost was equivalent to about US $\$ 6$ million which turns out to be 2.34 (or the aperture in meters!) times the original estimate.

\section{Other Reports}

Mr. Parn-an Shen, Nanjing Astronomical Instruments Factory, presented a summary concerning 16 major new facilities in China. These include a $1.26-\mathrm{m}$ infrared telescope and a $2.16-\mathrm{m}$ reflector for Beijing Observatory; a $1.56-\mathrm{m}$ astrometric telescope for Shanghai; a $0.35-\mathrm{m}$ solar magnetic field telescope for the Huairou Reservoir Station, Beijing; and a new solar fine-structure telescope for Yunnan Observatory. Several radio installations and a balloon-borne telescope were described.

$\mathrm{Mr}$. Chang-xin Cao, also at NAIF, considered the optimization of optical systems especially for large telescopes. Designs proceeding from collaborations with A. Meinel and R. N. Wilson were mentioned.

B. N. Karkera at Bhabhe Atomic Research in Bombay proposed a novel and simplified equatorial mount which consists of a cylindrical horse shoe with 5 oil pad bearings. Less than $50 \%$ as massive as conventional designs the advantage is in cost, stiffness, and ease of fabrication.

Albert Betz, University of California Space Sciences, described a farinfrared $(150 \mu$ to $500 \mu)$ laser heterodyne spectrometer for airborne astronomy. Operating above the tropopause, the instrument has been used to detect fine structure of $\mathrm{CI}$ in molecular clouds.

Multi-object Spectroscopy - 25 November

(Organizer: W. L. Wilcock; Chairman: C. M. Humphries)

Richard Green (KPNO): "Multi-object Spectroscopy with the Kitt Peak Cryogenic Camera and 4-meter Telescope." A computer planned photographic mask in the focal plane serves as entrance apertures (i.e., slits). Followed by a field lens, doublet collimator and a grating prism, resolutions of $8 \mathrm{~A}$ to $30 \mathrm{~A}$ are provided between $4900 \mathrm{~A}$ and $8000 \mathrm{~A}$. Detection is by a Texas Instrument 3-phase $800 \times 800$ thinned CCD after a demagnification of 8.4 . Imagery is at $\mathrm{f} / 1.0$. Typical format consists of 10-12 slits, each 10-15 arcseconds. Major limitation comes in sky subtraction because of focus variations arising from ripples in the thinned chip.

Donald Morton (AAT) "The Multi-object Fiber Spectroscopy System at the AAO."

Bernard Fort (Toulouse): "Multi-object Observations of Faint Objects at the CFHT with an On-1ine System, PUMA1."

Fred Watson (UKSTU): "The UK Schmidt Telescope 'FLAIR' Multi-object Spectroscopy System." $45 \mu$ fibers, glued to a positive copy glass plate, pick up objects over the 40 square degree FOV. These fibers feed a simple spectrograph with photographic film as the detector. Useful spectra are obtained to $\mathrm{V} \sim 14$. With a CCD detector the system should go to $\mathrm{V} \sim 17$.

Chris Impey (Caltech): "Radio Galaxies and Active Galaxies in Clusters" operating at the Cass focus of the 3.9-m AAT, 50 fibers of $200 \mu$ diameter are divided between objects and sky positions specified to 0.3 arcseconds. Centering and alignment takes about 5 minutes at the telescope. Detection is by the IPCS 
with a $2048 \times 200$ format corresponding to $0.88 \mathrm{~A}$ per channel. During a three-night run, over $65 \%$ of the time was spent in target integration. Results for cilustered radio galaxies were presented.

Keith Taylor (RGO): "A Low Dispersion Survey Spectrograph for the La Palma 4.2-m Telescope and The AAT."

Dave Carter and Peter Teague (Mt. Stromlo): "Velocity Structure of Clusters of Galaxies."

R. Foy (CERGA), A. Baranne (Marseille), and F. Thevenin (Nice): "The Large Multi-slit Spectrograph 'SFM'." An array of custom entrance slits are prepared on aluminized plates coated with photoresist. Matching the CFHT 15 areminute FOV of the Cass focus, a resolution $\sim 0.5 \mathrm{~A}$ from 3500 to $5500 \mathrm{~A}$ should be achieved. Guiding is by two field stars. Radial velocity is derived with aid of two comparison spectra and by inserting a 'lame en toit' spectrum divider. Aim is $\mathrm{V}-20$ in 5 hours for a $\mathrm{S} / \mathrm{N}=10$.

H Lorenz (GDR): "Multi-object Spectroscopy with the USSR 6-m Telescope." A multi-slit system is described which photographically records $200-250$ spectra per the 14 arcminute field. Recently an electronographic camera and a SIT TV have been used to record the spectra.

WG On Photoelectronic Image Devices - 22 November

(Chairman: Bernard Fort)

Gordon walker (Univ. B. C.): "On Choosing a Detector." There is still no a11-purpose detector. Choice must reflect the application and available funds. A full system consisting of detector, control electronics, data recording and display will cost about US $\$ 75,000$ whether built commercially or in the lab. Requirements for the differing applications were summarized. Present practical detector specifications were reviewed.

Richard Green (KPNO): "Overview of the New Tektronix CCDs." Tektronix is the successor to TI technology. Experimental $27 \mu$ pixel chips of size $512 \times 512$ $(13.8 \times 13.8 \mathrm{~mm})$ and $2048 \times 2048(55.3 \times 55.3 \mathrm{~mm})$ have been frabricated. Each chip has two serial registers: one for low-noise slow-scan and one for fast reading. A read noise of less than $10 \mathrm{e}$ is promised. Many specifications and results of tests were given but at this stage everything is preliminary. Such a large format will affect future instrument planning significantly.

R. Griffiths (STSCI), et al.: "An Advanced Radial Camera for the Hubble Space Telescope." The promise of Tektronix $2048 \times 2048$ arrays with $27 \mu$ pixels, low read noise and large full well capacity $\left(7 \times 10^{5} \mathrm{e}\right)$ suggest pursuing a second generation of cameras for the HST.

D. Thorne (RGO): "Experiments with the GEC/AAO 1500x1500 CCD."

D. Thorne (RGO): "Microchannel Plate Development at the RGO."

M. Cullum (ESO): "Photon-counting Systems vs. CCD's." It is pointed out that even with the availability of low read noise CCDs there is a domain of applications such as UV spectroscopy, speckle interferometers, and imaging FabryPerots where photon-counting wins out.

Richard Green (KPNO): "The Kitt Peak Photon-Counting Array." This is a more-or-less conventional system with a rapid scan detector which records intensified events and centrodes to a fraction of a pixel referred to the photocathode. First stage is a Carnegie 2-stage intensifier followed by an ITT 
dual microchannel plate tube. This is coupled to a Fairchild 222 CCD clocked at $20 \mathrm{mHz}$ yielding a $6 \mathrm{~ms}$ full frame time for a $3040 \times 976 \times 16$ bit accumulator. An option is dithering the first stage to suppress a centroiding fixed pattern.

A. Blezit and R. Foy (CERGA): "The "Photon-counting Camera CP40." Under development is a $2000 \times 2000$ pixel PCD for speckle and multislit spectroscopy. First stage is a $40 \mathrm{~mm}$ VARO intensifier followed by a $50 \mathrm{~mm}$ microchannel plate RTC intensifier. Four Thompson $288 \times 385$ CCDs are imaged in quadrants by a custom fiber-optic coupler. The accumulator size is then $3080 \times 2304$.

Craig McCreight (Ames Res. Cent.): "Infrared Astronomical Applications of Two-Dimensional Detector Arrays."

I. S. McLean (R. O. Edinburgh): "IR Imaging at the UKIRT." R. O. Edinburgh is developing imaging systems in the 1-5 $\mu$ region for use on the 3.8-m UKIRT. Capable of operating a variety of devices requiring different temperatures and control voltages, the first detector will be a SBRC $58 \times 62$ InSb direct readout device.

R. Puetter (San Diego): "Work on IR Array Detectors at the U.C.S.D." Two $\mathrm{Si}: \mathrm{Bi}$ detectors in the thermal IR $(5-18 \mu)$ are described. The first is a 128 linear array of $20 \times 6 \mathrm{mil}$ pixels on $8 \mathrm{mi}$ centers. Each pixel is connected to a $100 \mathrm{pF}$ capacitor so full well size of $10^{9} \mathrm{e}$ accommodates long exposures.

F. Lacombe, P. Lena, D. Rouan, and F. Sibille (Obs. de Paris et Lyon): "Ground-Based Imaging in the Near IR (1-5 $\mu)$ : Progress Report on a ChargeTransfer Array." (Presented by C. Cesarsky) A $32 \times 32$ InSb CID array has been manufactured for us by the French company SAT. The array has $100 \mu \times 100 \mu$ pixels with a fill factor of $80 \%$. Quantum efficiency in the middle of the $2-5 \mu$ region is $-50 \%$ and integrations of up to 100 seconds have been achieved at $4 \mathrm{~K}$. The readout noise is measured as $1200 \mathrm{e}^{-}$and the device does not exhibit much time lag or slow response. The CID array has already been used to obtain astronomical images at Pic du Midi. Jupiter and Saturn were imaged, both in broad-bands and in a methane absorption feature, with a spatial resolution of 2 ".

J. W. V. Storey, J. Karianski, S. T. Shanahan, M. A. Green, and U. Theden (U. New South Wales): "IR CCD Development at UNSW." Metals-silicide Schottky arrays for 1 to $6 \mu$ with CCD readouts are being tested on the AAT.

G. Wlerich (Obs. de Paris): "Observations avec la Camera Electronique Grand Champ au CFHT." In operation since December 1982, at the $\mathrm{f} / 8$ focus, the large ( $80 \mathrm{~mm})$ field of effectively $22 \times 10^{6}$ pixels permits full use of the excellent resolution as allowed by the telescope and site. Exposures up to 4 hours are effective. Application includes crowded field photometry and the study of small extended objects against foreground stars.

Abhijit Saha (KPNO): "A High Speed Plate Scanner with CCD Imaging and On-line Data Processing."

Harold Ables (USNO Flagstaff): "CCD Astrometry."

WG on Astronmical Photography - 22 November

Chairman: Elizabeth M. Sims

BUSINESS: Most of the discussion was based on the contents of a telex received from Eastman Kodak in response to questions from the WG Chairman about the supply, cost and availability of materials.

AVAILABILITY OF SPECTROSCOPIC EMULSIONS ON FILM: Technical Pan 2415 
emulsion may be available by the end of 1986 coated on to thick ( 0.7 mil) Estar base $\mathrm{film}$, in large formats, although the minimum order quantity is likely to be about 750 square feet. The production of other spectroscopic emulsions on thick base large format film is under consideration but there are as yet no plans to begin production. Many potential users welcomed the avallability of spectroscopic emulsion on film, and especially the price advantage over the same emulsion on glass, but it was also clear that a significant number of users still require glass plates for more critical applications.

COST OF PHOTOGRAPHIC PLATES: The large inerease in the cost of photographic material in recent years, made worse by the strong US dollar, has created grave problems for many users of spectroscopic plates. Small departments often find that they cannot afford even the modest number of plates they need. Unfortunately by commercial standards the astronomers are an extremely small fraction of the photographic market, and is only marginally profitable. Two approaches to this problem were agreed: (i) The incoming Chairman will write to Eastman Kodak Company expressing concern at the recent price increases and the consequent effects on astronomical research facilities; (ii) Users of spectroscopic materials are encouraged to collaborate in ordering materials, especially where several small orders can be combined into one larger one, thereby overcoming the minimum order constraints and costs. If timing orders to meet specified production dates, possibly twice a year, would help to keep costs down, many users seemed willing to fit in with such a time scheme.

ORDERING AND DELIVERY PROBLEMS: Many local Kodak agents do not know about spectroscopic materials, and consequently users experience difficulty in obtaining information, placing orders, and receiving shipments. In these circumstances help may be forthcoming from Nanci Snyder, of the International Division of Eastman Kodak Company, Rochester. In some circumstances, and with the agreement of the appropriate national Kodak agent, it may even be possible for users to place orders directly with Eastman Kodak International Division.

The new officers and Organizing Committee for the Working Group were agreed as follows: Chairman: D. F. Malin (Australia), Secretary: S. Marx (GDR); Organizing Committee: O. Dokuchaeva (USSR), J-L. Heudier (France), B. Hidayat (Indonesia), K. Ishida (Japan), A. G. Millikan (USA). W. E. Schoening (USA), J. G. Schumann (ERG), M. E. Sim (UK), K. R. Sivaraman (India), A. G. Smith (USA), M. K. Tsvetkiov (Bulgaria), R. M. West (FRG), and O. Zichova (Czechoslovakia). It was agreed that the name of the Working Group should become the "Working Group on Astronomical Photography." There is a proposal to hold another meeting of the Working Group, probably in Jena, GDR, in 1987.

Reports

J-L. Heudier, "Calibration and Sensitometry at TESCA." A Kitt Peak-style sensitometer is used to project a stepwedge onto each plate. After processing, the stepwedge density measures are used in a BASIC program to provide $H+D$ curve, DQE and $S: N$ curves for each plate automatically.

A. R. Good and M. E. Sim, "Microspots - A Progress Report." Further inspection of the plate collection reveals a higher than expected number of IV-N plates developing microspots.

J. Quebatte, B. Dumoulin, and R. M. West, "Grid Processing of Large Photographic Plates." This new method of developing large format plates gives more uniform and efficient results than the rotating rocker.

M. F. MeCarthy \& V. M. Blanco, "Detection of Carbon Stars - IIIa-J And IV-N Plates Compared." 
Syuzo Isobe and Hiroshi Maseda (Tokyo), "An Image Detection System Developed For Schmidt Plates With Large Numbers of Stars."

G. Wlerick, G. Lelievre, B. Servan, L. Renard, D. Horville and M. Poinse, "Emploi de la Camera Electronique A Grand Champ Avec Le Telescope CFH."

M. Capaccioli, "Calibration of Photographs." The quality of calibrations provided on photographic plates is sometimes reasonable, occasionally good, but usually very poor. Managers of telescope facilities are encouraged to make goodquality calibrations available more often.

It is still the policy of the Working Group to encourage authors to publish papers relating to astronomical photography in the AAS Photobulletin.

WG on High Angular Resolution Interferometry - 25 November (Chairman: J. Davis)

P. Nisenson, C. Papaliolios, S. Ebstein and R. Stachniki (CFA), "Center for Astrophysics Speckle Imaging Program." The program is built around two cameras: the PAPA (precision analog photon address) detector camera for low light level experiments and a Photometrics Inc., RCA-chip-based CCD camera for solar surface imaging. The CCD camera can be used in intensified mode for narrow spectral line work. The PAPA camera is a $512 \times 512$ element sensor which records photons in sequential format, with arrival times, at $\mathrm{mHz}$ rates. Decoding is done with the same electronics upon return to the laboratory. The Knox-Thompson procedure is used for image recovery. Recent results include detection of a 15 th mag. companion to 10 th mag. T Tau and a 5 th mag. companion to a Ori (separation: 0.05$)$.

J. E. Baldwin (Cambridge), "First Measurements of Closure Phase for Highresolution optical Imaging." As a first step in applying phase closure techniques developed for radio VLBI to optical wavelengths, short exposure CCD images have been recorded with the University of Hawail 88 inch telescope through aperture masks in the pupil plane. Each mask contained 3 or 4 holes corresponding to sizes in the aperture plane in the range $13-45 \mathrm{~cm}$. Fringes of high $v$ isibility were observed for single stars for exposures $t<50 \mathrm{~ms}$. Closure phases were derived for a variety of masks and exposure times. The results suggest that closure phases accurate to $<1^{\circ}$ should be possible after a few-minute observation. Further observations using photon-counting detectors have been made and are expected to give diffraction limited images of simple objects. The limiting magnitude of the technique is expected to be at least +10.5 and perhaps +15 depending on atmospheric conditions.

P. Venkatakrishnan \& S. Chatterjeel (IIA), "On the Saturation of the Refractive Index Structure Function at Large Separations: Enhanced Hopes for Long Baseline optical Interferometry." The conventional "2/3 power law" for the refractive index structure function is an approximation which is valid only in the inertial subrange of turbulence. For separations larger than the outer scale the structure function will tend to a constant value. A form for the structure function including saturation has been assumed to rederive the phase structure function following Roddier. This function tends to a constant at large separations and follows a power law with index 1.37 at small separations. Such a behavior of the structure function does not require the indefinite narrowing of bandwidth with increasing baseline and thus enhances the hopes for long baseline optical interferometry.

F. Vakili, Y. Rabbia \& L. Koechlin (CERGA, Saint Vallier, France), "Recent Results with $12 \mathrm{~T}$ in the Visible." The stellar interferometer at CERGA is currently working with a spectral range of $0.4-0.7 \mu$ and baselines from $6-67 \mathrm{~m}$ 
which provide angular resolution from 15-2 mas. Fringes are found in dispersed light using either low dispersion of $2 \mathrm{~nm} / \mathrm{pixel}$ with a total bandwidth of $200 \mathrm{~nm}$ or high dispersion of $0.5 \mathrm{~nm} / \mathrm{pixel}$ with a $30 \mathrm{~nm}$ bandwidth. Stellar images are superposed to better than $1 "$. The guiding $11 \mathrm{mit}$ is +8 mag using a photoncounting camera. Methods of fringe processing have been developed for the determination of angular diameters and for the automatic detection of fringes at low light levels (used to find high dispersion fringes on the +3.7 mag star $\lambda$ Lyr). The envelope around the shell star $\gamma$ Cas has recently been resolved using this device.

W. J. Tango and J. Davis (University of Sydney), "The University of Sydney Prototype Stellar Interferometer." A prototype stellar interferometer has been constructed as an essential step in the development of a very high angular resolution instrument. Except for a single baseline of $11.4 \mathrm{~m}$ and limited range optical delay lines, the prototype has all the essential features of the large interferometer. All the optics, including the fixed siderostats, are mounted on massive concrete supports to provide mechanical stability and active optical delay control is used. The effects of atmospheric turbulence are minimized by using apertures $\sim r_{0}$, active wavefront tilt correction and rapid data sampling. Interference was observed with starlight in October on the first observing night after alignment of the instrument was complete. The visibility maximum has been tracked for a number of stars (optical bandwith $0.3 \mathrm{~nm}$ ) allowing the baseline coordinates to be determined to $-0 ! 2$. The servo bandwidths, etc. are being optimized to maximize the detected fringe visibility and measurements on "standard" stars are planned to commence soon.

J. Davis \& W. J. Tango (University of Sydney), "The University of Sydney New High Angular Resolution Stellar Interferometer." Based on experience in developing the prototype interferometer, a new high angular resolution stellar interferometer has been designed. The new instrument will have a $\mathrm{N}-\mathrm{S}$ linear array of baselines covering the range $5-640 \mathrm{~m}$ which will permit angular sizes in the range 2-0.05 mas to be measured. Siderostats with $0.2 \mathrm{~m}$ diameter mirrors will give a primary beam diameter of $0.14 \mathrm{~m}$ and the limiting magnitude is expected to be -+8.5. The critical beam-combining optics from the prototype interferometer will be used in the new instrument. A proposal for funding has been submitted to the Australian Federal Government.

J. Hughes; (USNO), "The Mark III Astrometric Interferometer." Following the successful test program with Shao's Mark II astrometric interferometer the Mark III instrument is being constructed on Mt. Wilson. The new instrument features $10 \mathrm{~m}$ and $20 \mathrm{~m} \mathrm{~N}-\mathrm{S}$ baselines and $7 \mathrm{~m}$ and $14 \mathrm{~m} \mathrm{NE}-\mathrm{SE}$ baselines. Piers for the siderostats and the path compensation system have been poured and a temperaturecontrolled instrument laboratory has been constructed. Siderostats have been built at USNO with apertures of $0.25 \mathrm{~m}$. Corner cubes have been included in the back of the siderostat flats, as close to the front reflecting surface as possible, to permit their positions relative to the piers to be monitored with laser interferometers. The pier separation will also be monitored by laser interferometry. The project is a collaborative effort between SAO, MIT, USNO and USNRL.

W. Traub, M. Shao \& P. Nisenson (CFA), "The 40 Metre Imaging Stellar Interferometer on Mt. Wilson." It is proposed that an Imaging Stellar Interferometer (ISI) be built on Mt. Wilson with a $40 \mathrm{~m} \mathrm{~N}-\mathrm{S}$ variable baseline. It would have its own two $\sim 0.6 \mathrm{~m}$ diameter, collecting telescope, focal plane optics and control software but would time-share the variable delay line and star-tracker system from Shao's Mark III astrometric interferometer. The ISI would operate in three modes: (1) single $r_{0}$ to measure visibility amplitudes to 9 th mag; (2) as for (1) but with phase also measured; and (3) multi- $r_{0}$ to measure visibility amplitudes in photon-starved mode to about 13 th mag. The focal plane optics (including 
photon-counting camera) are being assembled and tested and the detailed design of the siderostats, variable basel ine, etc. will commence soon.

R. Stachnik \& W. Traub (CFA), "Report on the October 1985, Cambridge Space Imaging Interferometry Workshop." This meeting, convened by the astronomy subcommittee of a U.S. National Academy panel, developed recommendations to NASA on scientific and technological drivers for space imaging interferometry in the interval 1995-2015. Seven recommendations were formulated including: early development of critical technologies identified in the report, support for a precursor experiment, encouragement of ground-based efforts, long-term planning for a major observatory-class instrument, formation of a U.S. Working group analogous to the one in Europe and encouragement of the international efforts characterizing work in this area to date.

R. Stachnik \& M. Faucherre (CFA), "SAMSI, A Multiple Spacecraft Interferometer." SAMSI is a proposed space Michelson interferometer consisting of three spacecraft: two light collectors and a central beam-combiner. Detailed calculations indicate certain orbits are extremely favorable for efficient onedimensional surveys of angular diameters to 0.01 mas corresponding to a baseline of $10 \mathrm{~km}$. In a second observing mode, synthesis of filled apertures hundreds of metres in diameter would be possible on a timescale of about a day using $1 \mathrm{~m}$ collectors and $0.5 \mathrm{~N}$ thrusters. Numerical simulations have been undertaken to demonstrate the feasibility of image construction using a self-referencing scheme which relies on approximate determination of Fourier plane phase from information contained in the dispersed fringes and on optimal error distribution across the highly-overdetermined $\mathrm{u}-\mathrm{v}$ plane.

M. Faucherre, R. Stachnik \& W. Traub (CFA), "SPI, Space Platform Interferometer." The large and growing number of proposed space interferometry projects suggest the need for a relatively modest precursor experiment to test concepts and produce unique science. SPI would be a $10 \mathrm{~m}$ class baseline imaging device which might fly on either the Eureca or U.S. co-orbiting platform. Emphasis would be on measurements in the ultra-violet and on testing different approaches to image fourier transform phase recovery. A highiy desirable scientific goal would be synoptic imaging of several supergiants at 5 to 10 resolution elements across the surface over a wide range of wavelengths.

W. Traub, M. Lacasse \& N. Carleton (CFA), "COSMIC, A High Angular Resolution Imaging Array in Space." COSMIC is a proposed dilute-aperture direct-imaging telescope in space. Engineering studies at NASA-Marshall Space Flight Center and the Perkin-Elmer Corp. have shown COSMIC to be a good candidate for an early space interferometer, in part because it utilizes technology which is close to that currently available. COSMIC produces images using several identical afocal collecting telescopes, a central beam-combining telescope, a rigid support structure, internal path compensation, and a one or two-dimensional layout. Baselines on the order of $35 \mathrm{~m}$ are planned. Direct imaging has the advantage of a wide field of view and very faint limiting magnitudes. In its fail-safe mode, COSMIC can operate as a speckle imager. Current activities include laboratory simulations with six separator mirrors.

R. D. Reasenberg (CFA), "POINTS, A Smal1 Astrometric Optical Interferometer in Space."

Business: Matters discussed included future meetings and methods of improving communication between members of the Working Group. The Working Group's Organizing Committee for $1985-8$ is J. Davis (Chairman), G. P. Di Benedet to, R. Foy, R. D. Reasenberg, R. V. Stachnik \& C. H. Townes. 